\title{
Consumo alimentar de crianças de 6 a 18 meses em creches
}

\author{
Six to eighteen-month-old children's food intake in \\ day-care centers
}

\author{
Mônica Glória Neumann SPINELLI ${ }^{1,3}$ \\ Rita Maria Monteiro GOULART² \\ Arali Luiza Primo SANTOS² \\ Ludimila Di Carla GUMIERO ${ }^{2}$ \\ Cláudia Carvalheira FARHUD ${ }^{3}$ \\ Érica Barbosa de FREITAS ${ }^{3}$ \\ Ludmila Ferreira DANTAS ${ }^{3}$
}

\section{R E S U M O}

\section{Objetivo}

Verificar o consumo alimentar de crianças entre 6 e 18 meses e avaliar a adequação de nutrientes.

\section{Métodos}

O estudo foi realizado com 106 crianças em creches do Município de São Paulo. Para verificar o consumo alimentar utilizou-se o método de pesagem direta, durante cinco dias. Para análise dos macro e micronutrientes utilizaram-se as referências Organización Mundial de la Salud 1985 e Dietary Reference Intakes 1997, e para fibras adotou-se a recomendação da American Academy of Pediatrics 1993.

\section{Resultados}

O estudo revelou oferta insuficiente de energia, ferro, cálcio, vitamina A e fibras. A proteína e a vitamina C excederam as recomendações.

\section{Conclusão}

Nos moldes do programa atual, é necessário que a criança receba, no domicílio, uma refeição láctea e outra salgada para complementar principalmente cálcio, energia, ferro e fibras.

Termos de indexação: consumo alimentar, criança, creche, micronutrientes.

\footnotetext{
1 Secretaria da Assistência Social da Prefeitura Municipal de São Paulo.

2 Curso de Nutrição, Universidade São Judas Tadeu.

3 Curso de Nutrição, Universidade de Mogi das Cruzes. Av. Cândido Xavier de Almeida Souza, 200, 08780-911, Mogi das Cruzes, SP, Brasil. Correspondência para/Correspondence to: M.G.N. SPINELLI. E-mail: spinelli@usp.br
} 


\section{A B S T R A C T}

\section{Objective}

To verify the food intake of children from 6 to 18 months old and to evaluate nutrient adequacy.

\section{Methods}

This study was carried out with 106 children, in day-care centers from the city of São Paulo. Food intake was evaluated through food weighing during five days. The Organización Mundial de la Salud, 1985, Dietary Reference Intakes, 1997 and American Academy of Pediatrics, 1993 were used as references for the analysis of macronutrients, micronutrientes and fibers, respectively.

\section{Results}

The study revealed insufficient offering of energy, iron, calcium, vitamin A and fiber. Protein and vitamin C were over the recommended amounts.

\section{Conclusion}

The children must receive one milky and one salty meal at home in order to complement food given at centers, mainly regarding, calcium, iron, energy and fiber.

Index terms: food intake, child, day-care centers, micronutrients.

\section{N T R O D U Ç Ã O}

Na infância, a nutrição adequada é fundamental para garantir o crescimento e desenvolvimento normal da criança e a manutenção da saúde, e isto é particularmente verdade para os pré-escolares, os quais, além de serem biologicamente vulneráveis, constituem um dos grupos populacionais que mais necessitam de atendimento'.

Neste contexto, é importante destacar que vários estudos têm demonstrado deficiências de cálcio, ferro e energia na alimentação de crianças menores de sete anos, apesar do consumo protéico adequado ${ }^{2}$.

Em 1992, pesquisa realizada pela Secretaria de Saúde do Estado de São Paulo, com crianças menores de dois anos atendidas em unidades básicas de saúde, encontrou $56 \%$ de prevalência de anemia ${ }^{3}$.

A constipação intestinal também tem se tornado um problema comum na infância, pela inadequação do consumo de fibras, atingindo $25 \%$ das consultas de gastroenterologia pediátrica ${ }^{4}$.
Em relação às vitaminas, segundo estudos epidemiológicos, a deficiência de vitamina $A$ aumenta a gravidade e o risco das três doenças que mais ameaçam a vida das crianças nos países em desenvolvimento: infecções respiratórias, diarréia e sarampo ${ }^{5,6}$. A vitamina $C$ também tem sido considerada fator importante na alimentação infantil, pois estimula a absorção do ferro não-heme em até dez vezes?

O Programa de Alimentação para as Creches, de acordo com as diretrizes estabelecidas ${ }^{8}$, deveria atender $100 \%$ das recomendações nutricionais para as crianças que permanecem nas creches no período de 12 horas diárias, não havendo definição para aquelas com permanência em período menor.

Em razão da importância da alimentação no atendimento das necessidades nutricionais na infância, este trabalho se propôs a verificar o consumo alimentar de crianças entre 6 e 18 meses, freqüentadoras de creche, e avaliar a adequação de macronutrientes, das vitaminas $A$ e $C$, dos minerais cálcio e ferro e de fibras, em relação às recomendações nutricionais. 


\section{CASUÍSTICAE MÉTODOS}

Foram estudadas 106 crianças entre 6 e 18 meses, no período entre 2000 e 2001, em sete creches da Secretaria de Assistência Social de São Paulo (Freguesia do Ó). Na época do estudo, a região da Freguesia do Ó contava com 49 creches e 600 crianças menores de 18 meses matriculadas, o que representou uma amostra de um sétimo das creches e um sexto das crianças nesta faixa etária.

A escolha das creches foi feita considerando-se o número de crianças menores de 18 meses, o número daquelas que permaneciam em período integral na creche e a permissão da diretora para o desenvolvimento da pesquisa.

O número de participantes do estudo representa o total das crianças nessa faixa etária presentes nos dias da pesquisa.

Todas as preparações que faziam parte de cada refeição (desjejum, colação, almoço, lanche e jantar) foram pesadas antes de serem oferecidas às crianças. Após a refeição, foram pesados os restos, verificando-se a ingestão real da criança.

Estabeleceu-se o porcionamento padrão de cada preparação (média de três pratos), usado como referência para calcular a quantidade proporcional do resto.

Para a pesagem dos alimentos utilizou-se balança eletrônica com precisão de 5 gramas e capacidade máxima de 15 quilos. Para o cálculo do valor nutricional empregou-se a tabela de composição centesimal ${ }^{9}$, não sendo considerados os condimentos devido ao valor energético baixo e às quantidades de micronutrientes não significativas. Para o cálculo do consumo de fibras utilizou-se a Tabela de Composição de Alimentos ${ }^{10}$.

Para avaliar a adequação de macro e micronutrientes foram adotadas como padrão de referência a Organización Mundial de la Salud (OMS) de $1985^{11}$ e as Dietary Reference Intakes (DRI) de $1997^{12}$, respectivamente. Para avaliação da adequação de fibras foi utilizada a recomendação da American Academy of
Pediatrics de $1993^{13}$, de 0,5g por quilo de peso, considerando-se como parâmetro o peso médio para crianças $<9$ meses: $8,5 \mathrm{~kg}$, de 9 a 12 meses: 9,5kg e >12: $11,0 \mathrm{~kg}$, perfazendo uma recomendação média de 4,3g, 4,8g e 5,5g de fibras por dia, respectivamente.

As crianças foram divididas em três faixas etárias: 6 a 8 meses, 9 a 12 e $>12$ meses, em razão das faixas de recomendação nutricional.

Para o cálculo das médias e desvio-padrão foi empregado o programa estatístico Epi Info versão 6.

\section{RES U L T A D O S}

A distribuição das crianças segundo sexo e faixa etária (Tabela 1) mostrou maior prevalência de crianças $>12$ meses $(50,9 \%)$, com ligeiro predomínio do sexo feminino $(55,6 \%)$.

O consumo alimentar das crianças foi analisado para as diferentes faixas etárias, calculando-se valores médios, desvios-padrão e adequação percentual das dietas (Tabela 2).

Em relação ao consumo de energia, observou-se uma adequação de aproximadamente $50,0 \%$ em todas as faixas analisadas $(57,0 \%$ a $58,9 \%)$, apesar da oferta regular de alimentos açucarados tais como bolachas e sobremesas industrializadas (sagu, gelatina, pudim, doce em massa, mel, geléia). Uma hipótese que poderia explicar este resultado é o curto intervalo de tempo entre as ofertas das refeições, levando a um consumo reduzido em todas, além de o jantar oferecer baixa densidade energética por ser composto freqüentemente de sopa.

Resultados semelhantes foram encontrados por Zaccarelli (2001) ${ }^{14}$, no consumo de crianças de três creches da Prefeitura do Município de São Paulo. Cruz et al. (2001) ${ }^{15}$ encontraram adequação entre $62 \%$ e $66 \%$ no consumo energético de crianças freqüentadoras de creche no Piauí.

O consumo protéico ultrapassou as recomendações para todas as faixas estudadas 
(145,9\% a 189,6\%). A maioria dos trabalhos que avaliaram consumo de proteínas na alimentação infantil encontrou resultados semelhantes ${ }^{14,16}$.

O consumo de uma alimentação hiperprotéica poderia ser um fator de proteção para a desnutrição, desde que atendidas as recomendações de energia. Neste estudo, para todas as faixas etárias, os valores de energia provenientes de carboidratos e lipídios foram de cerca de $30 \%$ e $18 \%$, respectivamente, em relação à energia total recomendada e, portanto, inadequados, segundo recomendação da Sociedade Brasileira de Alimentação e Nutrição $(\mathrm{SBAN})^{17}$. Como os carboidratos e lipídios são nutrientes fornecedores de energia, por excelência, as proteínas excedentes poderiam estar sendo mobilizadas para este fim.

Os valores de ferro encontrados, 38,4\% a $52,2 \%$ das recomendações, corroboram o alto índice de anemia ferropriva encontrado entre crianças nesta faixa etária.

A adequação da ingestão de vitamina $C$ encontrada neste estudo nas três faixas de idade, em patamares acima de $100,0 \%$ das recomendações (111,8\% a $118,1 \%)$, é muito favorável para aumentar a absorção de ferro não heme da dieta, além de fortalecer o sistema imunológico da criança.

A freqüência de alimentos como abóbora, cenoura, mamão, entre outros, favoreceu uma oferta razoável da vitamina A (adequação entre $92,2 \%$ e $98,0 \%$ ). Outros estudos em São Paulo encontraram resultados semelhantes ${ }^{14,16,18}$. Em outras localidades foram encontrados valores inferiores, com cerca de 29,0\% e 57,5\% em Piauí e Minas Gerais, respectivamente ${ }^{15,19}$.

A adequação de cálcio variou entre 73,8\% e $114,5 \%$, mas é importante considerar que poderá haver uma maior oferta caso a criança receba uma refeição láctea no lar. De acordo com estudo realizado por Holland (1999)20, em creches do Município de São Paulo, da mesma região, as mães costumam oferecer pelo menos uma refeição láctea em casa, o que colaboraria para atingir as necessidades nutricionais diárias de cálcio das crianças maiores de 12 meses.

Tabela 1. Número e percentual de crianças segundo sexo e faixa etária. São Paulo, 2001.

\begin{tabular}{|c|c|c|c|c|c|c|}
\hline \multirow{3}{*}{ Faixa etária (meses) } & \multicolumn{4}{|c|}{ Sexo } & \multirow{2}{*}{\multicolumn{2}{|c|}{ Total }} \\
\hline & \multicolumn{2}{|c|}{$\mathrm{F}$} & \multicolumn{2}{|c|}{$M$} & & \\
\hline & $n$ & $\%$ & $\mathrm{n}$ & $\%$ & $n$ & $\%$ \\
\hline $6 \vdash 9$ & 11 & 57,9 & 8 & 42,1 & 19 & 17,9 \\
\hline $9 \vdash 12$ & 18 & 54,5 & 15 & 45,5 & 33 & 31,1 \\
\hline$\geq 12$ & 30 & 55,6 & 24 & 44,4 & 54 & 50,9 \\
\hline Total & 59 & 55,6 & 47 & 44,4 & 106 & 100,0 \\
\hline
\end{tabular}

Tabela 2. Valores médios $(\bar{X})$ desvios-padrão (DP) e adequação de nutrientes das dietas das crianças estudadas segundo faixa, etária. São Paulo, 2001

\begin{tabular}{|c|c|c|c|c|c|c|c|c|c|}
\hline \multirow{2}{*}{ Nutriente } & \multicolumn{3}{|c|}{6 a 9 meses } & \multicolumn{3}{|c|}{9 a 12 meses } & \multicolumn{3}{|c|}{12 a 18 meses } \\
\hline & $\bar{x}$ & $\mathrm{DP}$ & Adequação (\%) & $\bar{x}$ & $\mathrm{DP}$ & Adequação (\%) & $\bar{x}$ & DP & Adequação (\%) \\
\hline Energia (kcal) & $477,02 \pm$ & 76,26 & 58,9 & $559,95 \pm$ & 139,62 & 58,9 & $656,07 \pm$ & 111,99 & 57,0 \\
\hline Proteína (g) & $20,43 \pm$ & 3,58 & 145,9 & $22,64 \pm$ & 5,25 & 158,3 & $25,06 \pm$ & 2,67 & 189,6 \\
\hline Vitamina A (mcg) & $354,37 \pm$ & 265,11 & 92,2 & $367,43 \pm$ & 153,70 & 98,0 & $380,39 \pm$ & 185,64 & 95,1 \\
\hline Vitamina C (mg) & $41,35 \pm$ & 17,05 & 118,1 & $39,91 \pm$ & 11,51 & 114,0 & $44,72 \pm$ & 15,84 & 111,8 \\
\hline Ferro (mg) & $3,84 \pm$ & 0,81 & 38,4 & $4,59 \pm$ & 1,40 & 45,9 & $5,22 \pm$ & 1,41 & 52,2 \\
\hline Cálcio (mg) & $294,49 \pm$ & 35,05 & 109,1 & $309,21 \pm$ & 94,81 & 114,5 & $369,01 \pm$ & 66,16 & 73,8 \\
\hline Fibras Totais (g) & $3,16 \pm$ & 0,37 & 73,5 & $3,50 \pm$ & 0,34 & 72,9 & $3,85 \pm$ & 1,33 & 70,0 \\
\hline
\end{tabular}


O consumo de fibras atendeu cerca de dois terços das necessidades, podendo representar um risco para constipação intestinal, pois o consumo alimentar no domicílio normalmente não inclui alimentos ricos em fibras como frutas e verduras ${ }^{20}$. Este resultado pode ser conseqüência da baixa oferta de frutas e principalmente verduras, somadas a um maior porcionamento de caldo de feijão do que propriamente do grão.

É importante destacar a natureza das fibras alimentares, uma vez que exercem funções diferentes no organismo. As fibras solúveis, as quais tendem a retardar o esvaziamento gástrico e a absorção de nutrientes, foram encontradas em todas as faixas etárias e apresentam consumo médio de 1,13g. As fibras insolúveis, apenas parcialmente fermentadas no intestino grosso e com atuação mais restrita ao aspecto físico, diminuindo o tempo de trânsito do bolo alimentar no intestino, aumentando a massa fecal e a capacidade de ligar-se a determinados nutrientes e outros compostos presentes no intestino, atingiram o consumo médio de 1,5g.

\section{O N C LUS Ã O}

Os resultados obtidos com a avaliação do consumo alimentar das crianças desse estudo permitiram as seguintes conclusões: a vitamina $A$ atingiu patamares aceitáveis; não foram atendidas as recomendações diárias de energia, ferro e fibras em todas as faixas estudadas e as de cálcio na faixa etária de 12 a 18 meses. A ingestão de proteína e de vitamina $C$ excedeu às recomendações.

Como o horário de permanência da criança na creche (em média nove horas) não é suficiente para o atendimento total das recomendações nutricionais, faz-se necessário que a família assuma parte desta responsabilidade, oferecendo em casa ao menos uma refeição de sal completa (jantar), para complementar as recomendações de energia, ferro e fibra. Esse objetivo poderia ser alcançado através de orientação nutricional, pois a inserção da mãe no mercado de trabalho favorece a maior disponibilidade de alimentos no lar.

Com relação às recomendações de energia, as refeições oferecidas nas creches deveriam apresentar maior densidade energética, proveniente principalmente de carboidratos complexos e lipídios.

O programa de alimentação das creches deveria, ainda, incluir alimentos fortificados com ferro ou, pelo menos, o leite fortificado, para aumentar a oferta diária deste mineral, uma vez que esta estratégia tem se mostrado eficiente na diminuição da anemia ferropriva.

\section{A GRADECIMENTOS}

Os autores agradecem o apoio da nutricionista Edna Hitomi Sesoko da Secretaria de Assistência Social do Município de São Paulo, Freguesia do Ó, e dos funcionários das creches, que contribuíram para o desenvolvimento da pesquisa.

\section{REFERÊ NCIAS}

1. Amaral MFM, Morelli V, Pantoni RW, Rosseti-Ferreira UC. Alimentação de bebês e crianças pequenas em contextos coletivos: mediadores, interações e programas em educação infantil. Rev Bras Cresc Desenv Hum 1996; 6:19-33.

2. Briley ME, Roberts-Gray C, Rowe S. What can children learn from the menu at the child care center? J Community Health 1993;18:363-77.

3. Torres MAA, Sato K, Souza Queiroz SA. A anemia em crianças menores de 2 anos atendidas nas Unidades Básicas de Saúde no Estado de São Paulo. Rev Saúde Pública 1994; 28:290-4.

4. Loening BV. Encopresis and soiling. Pediatr Clin North Am 1996; 43:279-98.

5. Freeden $T R$, Sowell AL, Henning $K T$, et al. Levels and severity of measles. Am J Dis Child 1992;146:182-6. 
6. Hussey G, Klein M. Measles-induced vitamin A deficiency. Ann NY Acad Sci 1992; 669:188-96.

7. Cesar AT. O uso de ácido ascórbico no controle da deficiência de ferro, utilizando a estrutura do Programa de Merenda Escolar [dissertação]. São Paulo: Faculdade de Saúde Pública, Universidade de São Paulo; 1990.

8. Secretaria Municipal de Abastecimento. Programas de Alimentação do Município de São Paulo. São Paulo; 1996. p.25-36.

9. Instituto Brasileiro de Geografia e Estatística. Tabela de Composição de Alimentos. Estudo Nacional da Despesa Familiar. Rio de Janeiro; 1995.

10. Philippi ST. Tabela de composição de alimentos: suporte para decisão nutricional. Brasília; 2001.

11. Organización Mundial de la Salud. Necesidades de energía y proteínas. Ginebra; 1985. (Serie de Informes Técnicos, 724).

12. Dietary Reference Intakes: recommended intakes for individuals, vitamins, foods and nutrition board. Washington: National Academy of Sciences; 1997.

13. American Academy of Pediatrics. Committee on Nutrition. Carbohydrate and dietary fiber. In: Pediatric Nutrition Handbook. 3rd ed. Elk Grove Village: AAP; 1993. p.100-6.

14.'Zaccarelli EM. Avaliação do estado nutricional de crianças e estrutura de funcionamento de creches no Município de São Paulo [dissertação]. São Paulo:
Faculdade de Ciências Farmacêuticas, Universidade de São Paulo; 2001.

15. Cruz GF, Santos RS, Carvalho CMRG, Moita GC. Avaliação dietética em creches municipais de Terezina. Rev Nutr 2001;14(1):21-32.

16. Lopez Cardona CM. Avaliação de consumo alimentar de crianças freqüentadoras de creches municipais de São Paulo [dissertação]. São Paulo: Faculdade de Saúde Pública, Universidade de São Paulo; 1999.

17. Sociedade Brasileira de Alimentação e Nutrição. Aplicações das recomendações nutricionais adaptadas à população brasileira. São Paulo: Legis Suma; 1990.

18. Salay E, Carvalho JF. Nutritional value of nursery school meals served in Campinas city, Brazil. J Food Systems 1995; 8:175-86.

19. Magalhães P. Parâmetros nutricionais em pré-escolares de creches municipais de Viçosa - MG, relacionados com anemia e deficiência de Vitamina A [dissertação]. São Paulo: Faculdade de Ciências Farmacêuticas, Universidade de São Paulo; 1997.

20. Holland CV. A creche e seu papel na formação de práticas alimentares [dissertação]. São Paulo: Faculdade de Saúde Pública, Universidade de São Paulo; 1999.

Recebido para publicação em 14 de novembro de 2001 e aceito em 17 de outubro de 2002. 